\title{
Near-infrared Integral-Field Spectrograph (NIFS): An Instrument Proposed for Gemini
}

\author{
Peter J. McGregor, Peter Conroy, Gabe Bloxham \\ and Jan van Harmelen \\ Research School of Astronomy and Astrophysics, Institute of Advanced Studies, \\ Australian National University, Canberra, ACT 0200, Australia \\ peter@mso.anu.edu.au,conroy@mso.anu.edu.au, gabe@mso.anu.edu.au, \\ jan@mso.anu.edu.au \\ Received 1999 March 11, accepted 1999 July 15
}

\begin{abstract}
In late 1998 the International Gemini Project Office identified a need for a low cost, near-infrared spectrograph to be commissioned on the Gemini South telescope on the shortest possible timescale. In response, the Research School of Astronomy and Astrophysics of the Australian National University proposed to design, construct, and commission a near-infrared, integral-field spectrograph on Gemini. The science drivers and novel design of the Near-infrared Integral-Field Spectrograph (NIFS) are described in this paper. NIFS will achieve significant economies in cost and schedule in several ways:

- By addressing targeted science with high efficiency. NIFS will primarily target velocity measurements in galaxies to study the demographics of black holes in galactic nuclei and the evolution of structural properties in high redshift galaxies. However, NIFS will also be applied to a wide range of general astronomical topics, but these will not dictate the instrument design.

- By adopting a largely fixed-format design. A $3 \cdot 2^{\prime \prime} \times 3 \cdot 2^{\prime \prime}$ 'stair-case' integral field unit (IFU) will feed a near-infrared spectrograph with four fixed-angle gratings mounted on a single grating wheel. A single, fixed-format camera will form the spectral image on a $2048 \times 2048$ Rockwell HgCdTe HAWAII-2 array. Two-pixel spectral resolving powers of $\sim 5400$ will be achieved with complete wavelength coverage in each of the $J, H$, and $K$ photometric bands through 32 optimally sampled $0 \cdot 1^{\prime \prime}$ wide slitlets. The velocity resolution of $\sim 55 \mathrm{~km} \mathrm{~s}^{-1}$ will be sufficient to achieve the targeted science objectives, and will allow software rejection of $\mathrm{OH}$ airglow lines.

- By packaging the NIFS instrument within a duplicate of the Near-Infrared Imager (NIRI) cryostat. The NIRI cryostat, On-Instrument Wavefront Sensor (OIWFS), detector focusing mechanism, control system, and EPICS software will all be duplicated with only minimal change. Construction of the duplicate NIRI cryostat, OIWFS, and control system will be done by the University of Hawaii.
\end{abstract}

Keywords: instrumentation: detectors - instrumentation: spectrographs - techniques: spectroscopic

\begin{tabular}{ll} 
& \multicolumn{1}{c}{ Acronyms Used } \\
ALADDIN & Astronomical Large Area Detector Development on InSb \\
AO & adaptive optics \\
CC & components controller \\
DC & detector controller \\
EPICS & Experimental Physics Industrial Control System \\
GNIRS & Gemini Near-Infra-Red Spectrograph \\
HAWAII & HgCdTe Astronomical Wide-Area Infrared Imager \\
IFU & integral field unit \\
IOC & input-output controller \\
IS & instrument sequencer \\
NOAO & National Optical Astronomy Observatories \\
NIFS & Near-infrared Integral-Field Spectrograph \\
NIRI & Near-Infrared Imager \\
OIWFS & On-Instrument Wavefront Sensor \\
SBRC & Santa Barbara Research Center \\
SDSU & San Diego State University \\
VME & Versa Module Europe
\end{tabular}




\section{Introduction}

The potential cancellation of the clone of the Gemini Near-Infrared Spectrograph (GNIRS) for Gemini South led the International Gemini Project Office in late 1998 to consider alternative ways of achieving a near-infrared spectroscopic capability on this telescope as soon as possible after operational handover, which is expected to occur in the second half of 2001. The Research School of Astronomy and Astrophysics of the Australian National University has proposed to design, construct, and commission a fast-tracked, low-cost, near-infrared, integral-field spectrograph on Gemini. The aim is to provide an optimised near-infrared spectroscopic capability at the Gemini telescope on the shortest possible timescale and at modest cost. The design of the instrument is based on the premise that under these constraints the Gemini consortium will be best served by a targeted instrument which produces data of the highest quality for a restricted range of applications.

The Near-infrared Integral-Field Spectrograph to study the demographics of black holes in galactic nuclei and the evolution of structural properties in high-redshift galaxies. NIFS will also be applied to a wide range of general astronomy topics, but these will not dictate the instrument design. Economies the NIFS integral field unit (IFU) spectrograph in a duplicate of the NIRI cryostat, duplicating the NIRI On-Instrument Wavefront Sensor (OIWFS), and duplicating the NIRI control system and EPICS software with only minimal essential change. Further economies of cost and timescale will be achieved by restricting the IFU spectrograph to only two mechanisms, a duplicate of the NIRI focal-plane mask wheel and a grating wheel adapted from the NIRI beamsplitter wheel mechanism.

The NIFS science drivers and design are described in this paper. The NIFS proposal was recommended by the Gemini Instrument Forum at its March 1999 meeting and the Gemini Science Committee at its April 1999 meeting. The Gemini Board approved proceeding with the Conceptual Design Study at its May 1999 meeting.

\section{NIFS Overview}

NIFS will be used with the Gemini laser guide star adaptive optics (AO) system. The baseline design is shown in Figure 1. A $3 \cdot 2^{\prime \prime} \times 3 \cdot 2^{\prime \prime}$ 'staircase' IFU feeds a near-infrared spectrograph with four fixed-angle gratings mounted in a grating wheel. A (NIFS) will target velocity measurements in galaxies of cost and timescale can be realised by packaging

single, fixed-format camera forms the spectral image on a $2048 \times 2048$ Rockwell HAWAII-2 HgCdTe array. Spectral resolving powers of $\sim 5400$ will be achieved with complete wavelength coverage in each of the $J, H$ and $K$ photometric bands through 32 fully sampled $0 \cdot 1^{\prime \prime}$ wide slitlets. The velocity resolution of $\sim 55 \mathrm{~km} \mathrm{~s}^{-1}$ is sufficient to achieve the targeted science objectives, and will allow software rejection of $\mathrm{OH}$ airglow lines.

Light enters at the left of Figure 1 through the dewar window and comes to focus $300 \mathrm{~mm}$ inside the dewar. The $3 \cdot 2^{\prime \prime} \times 3 \cdot 2^{\prime \prime}$ field passes to an Offner relay, which reimages the telescope exit pupil on a small secondary mirror that acts as a cold stop to baffle the system. The $f / 16$ beams from the Offner relay pass through a focal ratio converter which forms an $f / 90$ focus on an $11 \mathrm{~mm} \times 11 \mathrm{~mm}$ reflective image slicer (see Section 5.2) and also forms an $f / 90$ pupil further downstream. The image slicer directs each slice of the focal plane to a different element of the $f / 90$ pupil mirror array, where the 32 spherical mirrors reimage the $f / 16$ focal plane in the form of a $64 \mathrm{~mm}$ long 'staircase' slit on the field mirror array. The 32 spherical field mirrors are decentred by increasing amounts so that they feed telecentric beams (i.e. parallel cones) into the spectrograph. The spectrograph uses a doublet field lens to direct these beams towards a $30 \mathrm{~mm}$ diameter pupil on the grating at lower right in Figure 1 via a folding mirror and a doublet collimator lens. A folded camera views the diffracted light with a $30^{\circ}$ Ebert angle and focuses spectra from the 32 slitlets onto the detector at lower left in Figure 1.

\section{NIFS Science Drivers}

The primary science drivers for NIFS are the study of the demographics of massive black holes in the nuclei of galaxies, and the study of the dynamical evolution of galaxies at high redshift.

Table 1. Massive black hole galaxies

\begin{tabular}{lcclr}
\hline Galaxy & $\begin{array}{c}D \\
(\mathrm{Mpc})\end{array}$ & $\begin{array}{c}M_{B} \\
(\mathrm{mag})\end{array}$ & $\begin{array}{l}M_{B H} \\
\left(M_{\odot}\right)\end{array}$ & Ref. \\
\hline M 32 & $0 \cdot 7$ & $-15 \cdot 51$ & $3 \times 10^{6}$ & 1,2 \\
NGC 4486B & 20 & $-17 \cdot 15$ & $1 \times 10^{7}$ & 3,4 \\
Milky Way & - & $-17 \cdot 65$ & $2 \cdot 4 \times 10^{6}$ & 5 \\
M 31 & $0 \cdot 7$ & $-18 \cdot 82$ & $3 \times 10^{7}$ & 6 \\
NGC 3377 & 14 & $-19 \cdot 74$ & $1 \cdot 8 \times 10^{8}$ & 7 \\
NGC 3115 & 10 & $-20 \cdot 46$ & $2 \times 10^{9}$ & 8 \\
NGC 4258 & 9 & $-20 \cdot 46$ & $7 \times 10^{7}$ & 9 \\
NGC 4374 & 27 & $-21 \cdot 42$ & $1.5 \times 10^{9}$ & 10 \\
M 87 & 20 & $-21 \cdot 86$ & $3 \times 10^{9}$ & 11 \\
NGC 4261 & 30 & $-21 \cdot 87$ & $9 \times 10^{8}$ & 12 \\
NGC 4594 & 16 & $-23 \cdot 14$ & $1 \times 10^{9}$ & 13 \\
\hline
\end{tabular}

References: 1. Bender et al. (1996) 2. van der Marel et al. (1997) 3. Kormendy et al. (1997) 4. Lauer et al. (1996) 5. Eckart \& Genzel (1997) 6. Kormendy \& Richstone (1995) 7. Kormendy et al. (1998) 8. Kormendy et al. (1996a) 9. Miyoshi et al. (1995) 10. Bower et al. (1998) 11. Ford et al. (1994) 12. Ferrarese, Ford, \& Jaffe (1996) 13. Kormendy et al. (1996b). 


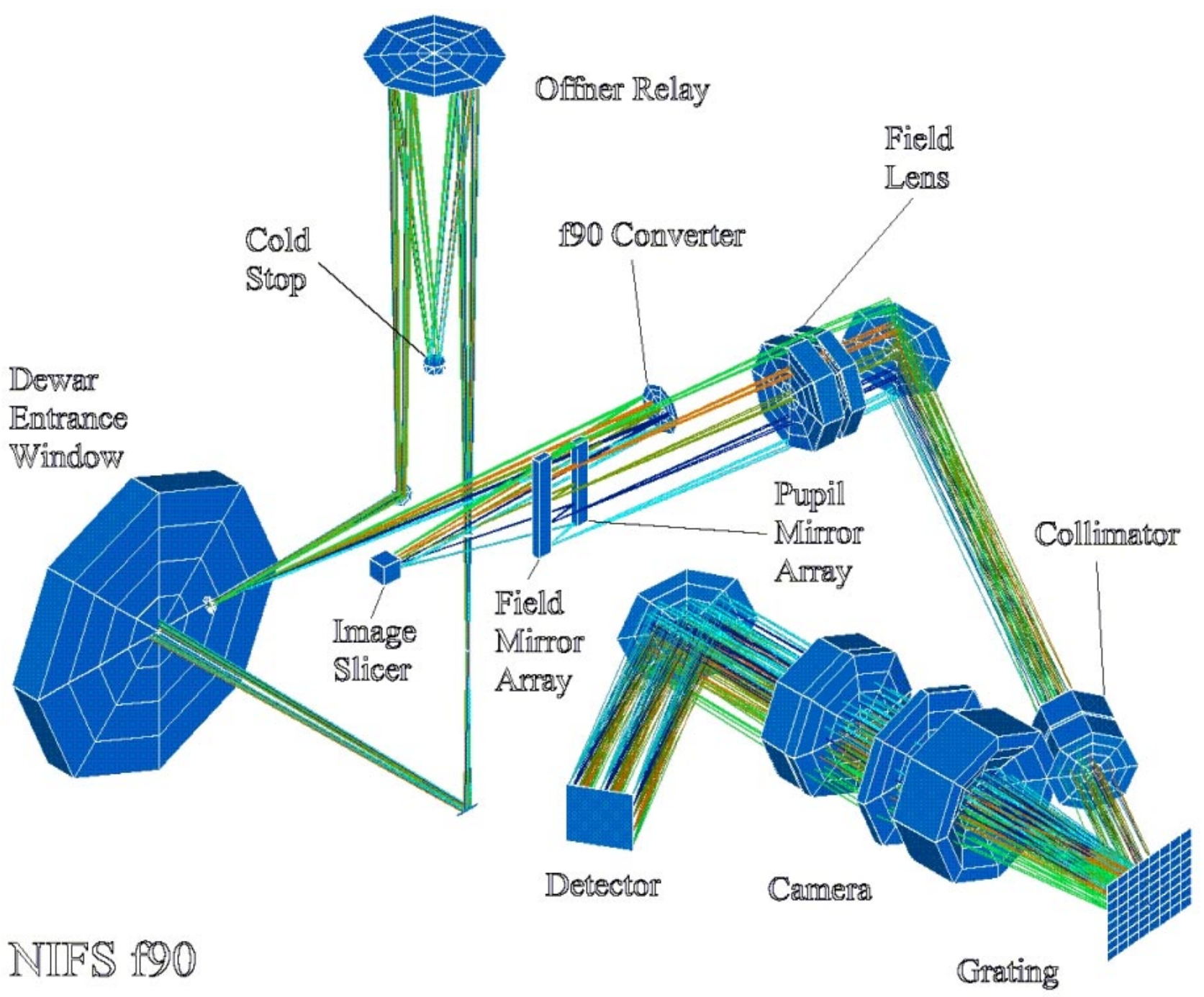

Figure 1-NIFS baseline optical configuration. The dewar window is shown at left. After passing through an Offner relay, the $f / 16$ beam is converted to $f / 90$ and reimaged onto the image slicer. This is followed by the pupil mirror array and the field mirror array of the IFU which feed the spectrograph. The grating is at lower right. The detector is at lower left.

\subsection{Massive Black Holes in Galactic Nuclei}

One of the most profound results from the Hubble Space Telescope (HST) is the evidence for the existence of massive $\left(10^{7}\right.$ to $\left.10^{9} M_{\odot}\right)$ black holes in the nuclei of many nearby galaxies (see Table 1, derived from Franceschini, Vercellone \& Fabian 1998). The mass distribution and frequency of occurrence of these central black holes is still poorly known (Kormendy \& Richstone 1995): understanding the demographics of these massive black holes is one of the most significant astrophysical problems we currently face.

Spatially resolved, high-resolution dynamical studies of the innermost nuclear stellar populations at near-infrared wavelengths are needed to progress these studies. Observations of surface brightness distributions, mean rotation, and radial velocity dispersion profiles, with spatial resolution of a few parsecs and spectral resolution of 3000 to 5000, are required to model the stellar dynamics and infer properties of the central black hole.
The aperture of HST is too small to make a definitive study of central black holes due to its limited light gathering power; the aperture of an 8$\mathrm{m}$-class telescope is needed, with $\mathrm{AO}$ resolution. The central regions of most galaxies contain obscuring dust, which complicates the interpretation of optical data from HST. Near-infrared data are essential. A bright, compact nuclear emission-line core very often contaminates direct imaging and spectroscopy of the inner stellar population, particularly in spiral galaxies. An occulting disk may be required to suppress contamination from these nuclei. In summary, a near-infrared integral-field spectrograph with an AO system and occulting disk capability is the best way to make a definitive study of central black holes in the decade before the Next Generation Space Telescope. The five essential elements for this work are the large aperture, the AO system, the integral-field spectroscopic capability, the occulting disk capability, and the near-infrared wavelength coverage. 
The dynamical mass $M_{\text {dyn }}$ enclosed within a radius $R$ can be estimated from the first moment of the collisionless Boltzmann equation (Sargent et al. 1978). For isotropic, isothermal stellar cores we have

$$
\begin{aligned}
& M_{\mathrm{dyn}}=\frac{2 \sigma_{*}^{2} R}{G} \\
& \approx 3 \times 10^{7}\left[\frac{R}{0 \cdot 5^{\prime \prime}}\right]\left[\frac{D}{10 \mathrm{Mpc}}\right]\left[\frac{\sigma_{*}}{50 \mathrm{~km} \mathrm{~s}^{-1}}\right]^{2} M_{\odot},
\end{aligned}
$$

where $\sigma$ is the radial component of the stellar velocity dispersion, $G$ is the gravitational constant, and we assume that the stellar radial velocity dispersion is constant over the region of interest and that the mass density distribution is $\rho(R) \propto r^{-2}$, as found for 13 spiral galaxies by Devereux, Becklin \& Scoville (1987). With a velocity resolution of $55 \mathrm{~km} \mathrm{~s}^{-1}$, NIFS should be able to measure velocity dispersions of this order, and so be capable of detecting mass concentrations of $\sim 3 \times 10^{7} M_{\odot}$ within $0 \cdot 5^{\prime \prime}$ of the centres of galaxies at distances of $\sim 10 \mathrm{Mpc}$.

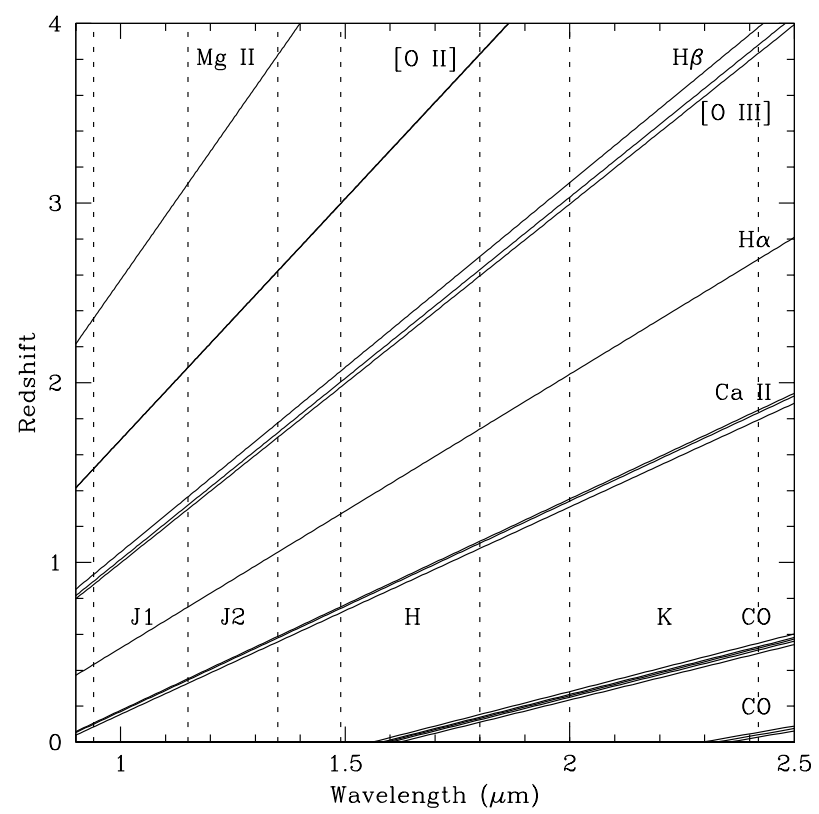

Figure 2-Wavelengths of strong absorption and emission features as functions of redshift. Features plotted are [O II] $0.3727 \mu \mathrm{m}, \mathrm{H} \beta 0.4861 \mu \mathrm{m}$, [O III] 0.4959,0.5007 $\mu \mathrm{m}$, $\mathrm{H} \alpha 0 \cdot 6563 \mu \mathrm{m}$, Ca II triplet $0 \cdot 8498,0 \cdot 8542,0 \cdot 8662 \mu \mathrm{m}$ in the rest-frame optical, CO (3-0), (4-1), (5-2), (6-3) and Si I $1.589 \mu \mathrm{m}$ in the rest-frame $H$ band, and CO $(2-0)$, $(3-1),(4-2)$ in the rest-frame $K$ band. Regions measured by the four NIFS gratings are indicated by dashed lines.

The CO (2-0) absorption bandhead at $2 \cdot 294 \mu \mathrm{m}$ is ideal for measuring stellar velocity dispersions in low-redshift galaxies. This band is strong in late-type stellar spectra and will be accessible with NIFS to a redshift of $\sim 0.05$ (Figure 2). The photometric $H$ band contains the second overtone CO absorption bands $[(3-0)$ at $1.56 \mu \mathrm{m},(4-1)$ at
$1.58 \mu \mathrm{m},(5-2)$ at $1.60 \mu \mathrm{m},(6-3)$ at $1.62 \mu \mathrm{m}]$ and the $\mathrm{Si}$ I $1.589 \mu \mathrm{m}$ atomic absorption line. These features will be accessible with NIFS to a redshift of $\sim 0 \cdot 1$ (Figure 2). Typical central $K$-band surface brightnesses have been estimated from aperture photometry and growth curves for $\sim 50$ bright early-type spiral galaxies (Griersmith, Hyland \& Jones 1982). In a $3 \cdot 2^{\prime \prime}$ diameter aperture, the average $K$-band surface brightness is typically $13 \cdot 7 \mathrm{mag} \operatorname{arcsec}^{-2}$ (Figure 3 ).

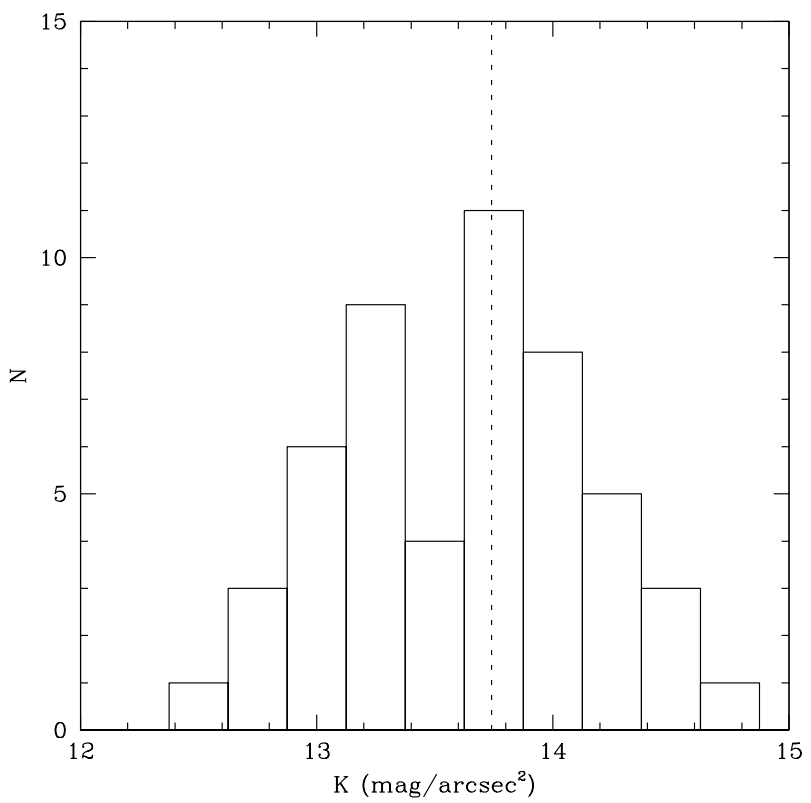

Figure 3-Histogram of central $K$-band surface brightness inferred from aperture photometry for $\sim 50$ bright early-type spiral galaxies (Griersmith et al. 1982). The average value of $13.7 \mathrm{mag} \operatorname{arcsec}^{-2}$ is indicated by a dotted line.

\subsection{Dynamical Evolution of Galaxies}

Understanding the formation histories of galaxies will arguably be one of the most important astronomical legacies of our generation, and is certainly one of the main justifications for constructing the Gemini telescopes. Massive elliptical galaxies seemed to have formed early in the universe at redshifts $z>2$. However, there is much theoretical and observational evidence that spiral galaxies formed over a long period of cosmic time extending to redshifts $z<1$. How spiral galaxies accumulated their material in the redshift range $1 \leq z \leq 2$ is one of the most important problems of observational cosmology, and one that will be tractable with NIFS.

Integral-field spectroscopy of high-redshift galaxies will provide fundamentally new and more precise information about the evolution of star-forming disk galaxies with look-back time. Measurements of disk rotation velocity with resolutions of $\sim 50 \mathrm{~km} \mathrm{~s}^{-1}$ at galactocentric radii of order 1 disk scale length will provide kinematic estimates of the total galaxy mass, and hence probe the mass assembly history of disk galaxies. Galaxy luminosities combined with 
assumptions about stellar mass-to-light ratios will allow crude separation of the luminous and dark matter components, and so provide information on the separate mass accumulation histories of these components and on the importance of biasing. $\mathrm{H} \alpha$ spectroscopy produces the best emission-line rotation curves, but $\mathrm{H} \alpha$ passes into the near-infrared region at redshifts $z>0 \cdot 5$. With near-infrared spectroscopy, $\mathrm{H} \alpha$ will be accessible from $z \sim 0.5$ to $z \sim 1.0$ in the $J$ band, $z \sim 1.3$ to $z \sim 1.7$ in the $H$ band, and beyond $z \sim 2$ in the $K$ band (Figure 2 ), well beyond $z \sim 1$ where current galaxy formation models predicted that present-day galactic disks were still forming. $\mathrm{H} \beta$ can be measured from $z \sim 1.0$ to $z \sim 1.7$ in the $J$ band at redshifts where $\mathrm{H} \alpha$ is not accessible from the ground. Integrated $\mathrm{H} \alpha$ and $\mathrm{H} \beta$ luminosities of disk galaxies provide instantaneous total star formation rates, which can be compared with the inferred mass accumulation rate, and integrated over cosmic time to constrain possible star formation histories.

For Canada-France Redshift Survey (CFRS) galaxies with $z \leq 0 \cdot 3, L_{\mathrm{H} \alpha}^{*}=10^{42 \cdot 13 \pm 0 \cdot 13} \mathrm{erg} \mathrm{s}^{-1}$, and $\mathrm{H} \alpha$ luminosity is related to absolute blue magnitude by $M\left(B_{A B}\right)=46 \cdot 7-1 \cdot 6 \log L(\mathrm{H} \alpha)$ (Tresse $\&$ Maddox 1998). The $B$ luminosity function for blue CFRS galaxies brightens by $\sim 1$ mag between $z \sim 0.3$ and $z \sim 1$ (Lilly et al. 1995). If $M\left(B_{A B}\right)$ is related to $L(\mathrm{H} \alpha)$ at $z \sim 1$ as it is at $z \leq 0 \cdot 3$, then $L_{\mathrm{H} \alpha}^{*} \sim 10^{42 \cdot 73}$ erg $\mathrm{s}^{-1}$ at $z \sim 1$. McCarthy et al. (1999) find a median $L(\mathrm{H} \alpha)$ at $0.75<z<1.9$ of $10^{42 \cdot 43} \mathrm{erg} \mathrm{s}^{-1}$ from slitless grism spectroscopy with NICMOS. We adopt this value. With $H_{0}=50 \mathrm{~km} \mathrm{~s}^{-1} \mathrm{Mpc}^{-1}$ and $q_{0}=0 \cdot 5$, a typical galaxy at $z \sim 1$ would then have an integrated $\mathrm{H} \alpha$ flux of $\sim 4.6 \times 10^{-23} \mathrm{~W} \mathrm{~cm}^{-2}$. Typical disk scale lengths are $\sim 4 \mathrm{kpc}$ (Schade et al. 1995), corresponding to a half-light radius of $\sim 0 \cdot 77^{\prime \prime}$ on the sky. If the $\mathrm{H} \alpha$ emission is uniformly distributed across the galaxy disk, the $\mathrm{H} \alpha$ surface brightness will be $\sim 2.5 \times 10^{-23} \mathrm{~W} \mathrm{~cm}^{-2} \operatorname{arcsec}^{-2}$.

\subsection{Secondary Science Opportunities}

The instrumental capabilities of NIFS for its primary science goals are common to a wide range of other science. Indeed NIFS will be able to perform much of the science planned for GNIRS on Gemini North and for the deployable IFUs proposed for the Gemini near-infrared multi-object spectrographs, albeit by measuring these objects sequentially. These secondary science opportunities include:
- high-resolution near-infrared spectroscopy of individual stars and substellar objects;

- studies of the dynamics and excitation of premain-sequence star disks;

- studies of the inner regions of pre-main sequence star jets;

- dynamical and abundance investigations of stellar populations in galaxies, including the centre of our galaxy;

- investigation of the dynamics, excitation, and abundances of gas in the inner narrow-line regions of nearby active galactic nuclei;

- dynamical, excitation, and abundance studies of the cores of starburst galaxies and ultra-luminous IRAS galaxies;

- observational confirmation of the existence of massive central black holes in the host galaxies of QSOs through direct measurement of high stellar velocity dispersions;

- study of stellar and gaseous dynamics and excitation in radio galaxy hosts, and the origin of radio jet/host galaxy alignment at high redshift.

\section{The NIRI Legacy}

\subsection{NIRI Cryostat}

The NIFS layout (Figure 1) is based on the realisation that significant cost savings will arise from packaging NIFS in an identical container to that used for NIRI (Young et al. 1998). NIRI ${ }^{1}$ is the nearinfrared imager being constructed by the University of Hawaii for Gemini North. It uses a cylindrical dewar $^{2}$ with a hexagonal cross section, mounted transverse to the optical axis. A 40-mm-thick 6061T6 aluminium optical bench is mounted inside the dewar on titanium struts. The NIRI OIWFS ${ }^{3}$ is mounted on one side of this optical bench, while the NIRI camera ${ }^{4}$ is mounted on the other side. We propose duplicating the NIRI cryostat and all NIRI components on the OIWFS side of the optical bench, except the NIRI beamsplitter wheel. The beamsplitter wheel will be replaced by a fixed mirror which directs the fixed-format science field to the camera side of the optical bench. The NIFS IFU spectrograph will replace the NIRI camera on this face (Figure 4).

This packaging solution permits fast-tracking of much of the NIFS construction, as well as providing a proven design for the OIWFS. The NIRI focal plane mask wheel will provide a convenient location at the telescope focal-plane for inserting the occulting spots which may be required to probe galactic black holes

\footnotetext{
${ }^{1}$ http://kupono.ifa.hawaii.edu/WEB/NIRI/NIRI1.html

${ }^{2}$ http://kupono.ifa.hawaii.edu/WEB/NIRI/mech/vj.html

${ }^{3}$ http://kupono.ifa.hawaii.edu/WEB/NIRI/mech/wfs-image.html

${ }^{4}$ http://kupono.ifa.hawaii.edu/WEB/NIRI/mech/cam-image.html
} 

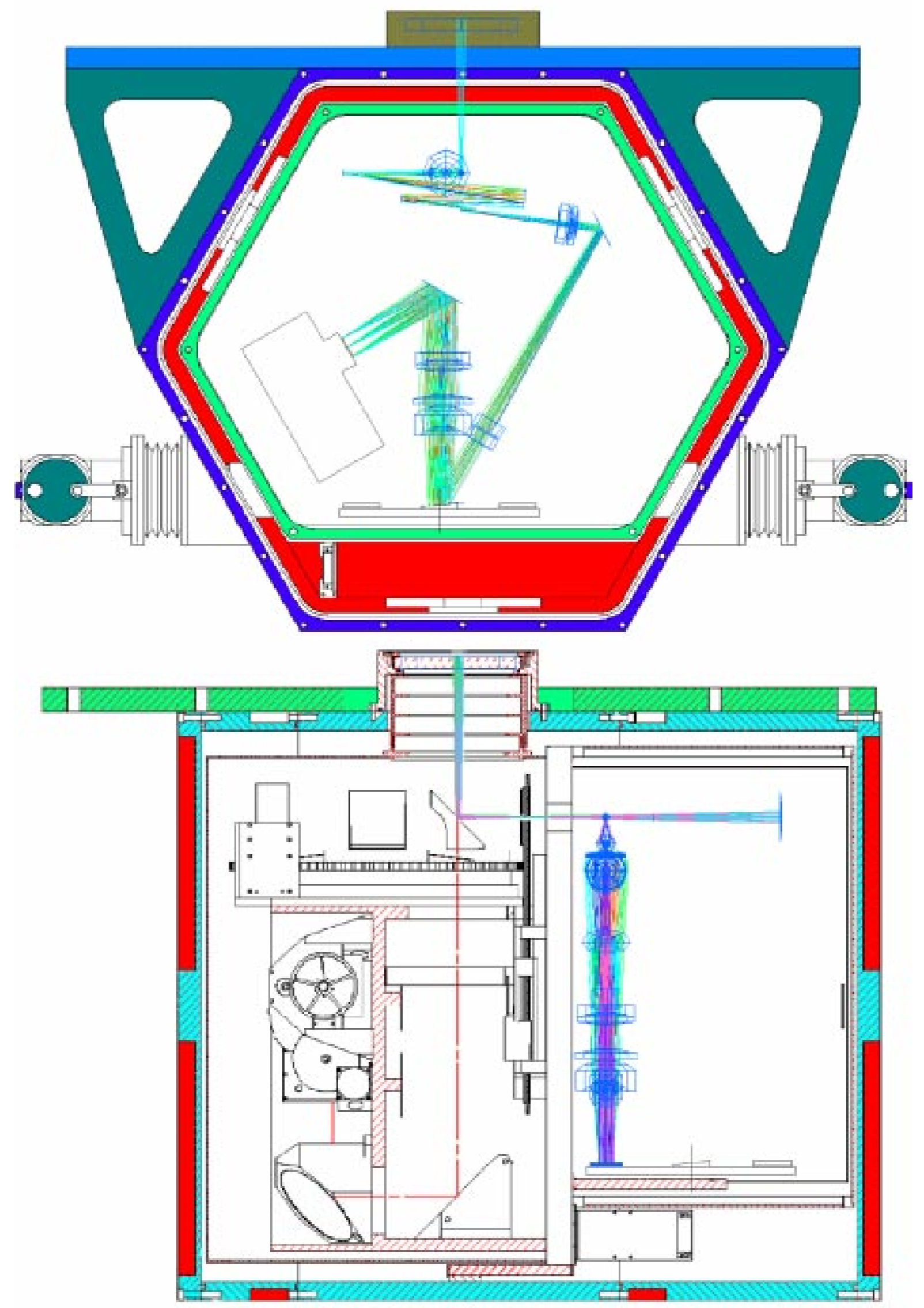

Figure 4-Schematic showing the NIFS optics located within the NIRI dewar. 
on small scales. The detented Geneva drive used for the NIRI beamsplitter wheel will be adapted to the NIFS grating wheel. The NIRI science detector focusing mechanism and the NIRI structures for mounting associated control electronics will also be duplicated for NIFS. Duplicate NIRI components will be constructed in collaboration with the University of Hawaii.

\subsection{NIRI Control System}

The wholesale adoption of much of NIRI means that the NIRI mechanism and temperature control systems $^{5}$ can also be duplicated to control NIFS with only relatively minor modification. NIFS will use the same Phytron cryogenic stepper motors used in NIRI and the same Hall effect sensors used to encode the NIRI mechanisms. NIFS will have fewer mechanisms in the science instrument, leading to a slight reduction in the control system complexity. The OIWFS will use a Rockwell ${ }^{6} 1024 \times 1024$ detector array and an SDSU- $2^{7}$ array controller, as does NIRI.

The main area in which the control systems for NIFS and NIRI differ is the science detector controller. NIRI uses a $1024 \times 1024$ SBRC ALADDIN array and the NOAO Wildfire detector controller. NIFS will use a $2048 \times 2048$ Rockwell HAWAII-2 array and an SDSU-2 detector controller with coadder boards. The SDSU-2 controller includes a standard VME interface to the detector input-output controller (IOC).

\subsection{NIRI EPICS Software}

The close similarity with the NIRI control system means that the EPICS ${ }^{8}$ software developed for NIRI will be directly applicable to NIFS with only minimal modification. The OIWFS component controller (CC) and detector controller (DC) will be directly applicable to NIFS. The NIRI engineering software interface, instrument sequencer (IS), and science instrument $\mathrm{CC}$ will require only minor modification to remove mechanisms and rename the grating wheel components.

The major EPICS software development envisaged is in the science instrument DC which will interface to an SDSU-2 controller. EPICS code may be available for aspects of this development through the NOAO workpackage to deliver SDSU-2 controllers for optical CCDs.

\section{NIFS Design Concept}

The NIFS optical design has been introduced in Section 2. We now describe each section of the instrument in greater detail.

\subsection{Offner Relay}

The Offner relay (Offner 1975) is a simple, highperformance system for reimaging a narrow field at the same scale while forming a real pupil that can be used to baffle the system. A conventional spectrograph forms a pupil on the grating. However, it is difficult to use a grating pupil as a cold stop in a near-infrared spectrograph. The Offner relay provides a convenient solution because the IFU requires only a small field.

\subsection{Integral Field Unit Specification}

The first near-infrared IFU spectrograph was the 3D instrument (Weitzel et al. 1996) constructed by the Max Planck Institut für Extraterrestrische Physik and used recently on the Anglo-Australian Telescope. A limitation of this instrument is that each reformatted slitlet is reimaged to only one detector pixel. A mechanism for half-stepping the grating is then required to fully sample the spectrum. NIFS will avoid this complication by mapping each slitlet to two detector pixels in the spectral direction. The $2048 \times 2048$ pixel detector array then accommodates 32 slitlets, each $0 \cdot 1^{\prime \prime}(2$ pixels) wide by $3 \cdot 2^{\prime \prime}$ (64 pixels) on the sky. The field of view of the IFU is $3 \cdot 2^{\prime \prime} \times 3 \cdot 2^{\prime \prime}$. An effective slit width of $0 \cdot 1^{\prime \prime}$ was chosen in order to probe to spatial scales approaching the $0 \cdot 07^{\prime \prime}$ diffractionlimited image core size expected from the Gemini $\mathrm{AO}$ system at $2 \cdot 2 \mu \mathrm{m}$.

\subsection{Integral Field Unit Philosophy}

The IFU is the most critical NIFS component. The reflective 'staircase' IFU approach is favoured over optical fibres, for example, because it is proven technology requiring the least development investment. Optical fibre solutions are complicated by the need to feed fibres with fast beams. Reflective IFUs are intricate devices, and some explanation is required to demonstrate how the NIFS IFU functions, and why a superior design can be achieved by mating it to a dedicated spectrograph, rather than retrofitting an IFU to an existing spectrograph.

The first 'staircase' IFU was used in the 3D instrument (Figure 5). This uses a 16-element reflective image slicer in which each of the flat mirrors is tilted at the appropriate angle to form the virtual slit. A 16-element segmented mirror then feeds light from each virtual slitlet into the spectrograph. The requirements that the virtual slit be flat and that the virtual pupil match the telescope exit pupil required that the flat segments of the segmented mirror be positioned along a

\footnotetext{
${ }^{5}$ http://kupono.ifa.hawaii.edu/WEB/NIRI/electronics/niriblk2.pdf

${ }^{6} \mathrm{http://www.rsc.rockwell.com/mct.fpa/}$

${ }^{7}$ http://mintaka.sdsu.edu/ccdlab/LabMain.html

${ }^{8}$ http://www.aps.anl.gov/asd/controls/epics/EpicsDocumentation/WWWPages/
} 
hyperbola. This simple design works well for small IFUs and small detectors. However, the long virtual slit possible with a $2048 \times 2048$ detector array means that the distance from the slicer to the extremity of the segmented mirror becomes large and the $f / 16$ beams from the Gemini telescope diverge to a size larger than each mirror segment. Specifically for NIFS, over the required distance of $32 \mathrm{~mm}$ the $f / 16$ beams would diverge to a width of $2 \mathrm{~mm}$. The $2 \mathrm{~mm}$ long slitlets would then fill $4 \mathrm{~mm}$ at the segmented mirror, overfilling their respective segmented mirror elements by a factor of two.

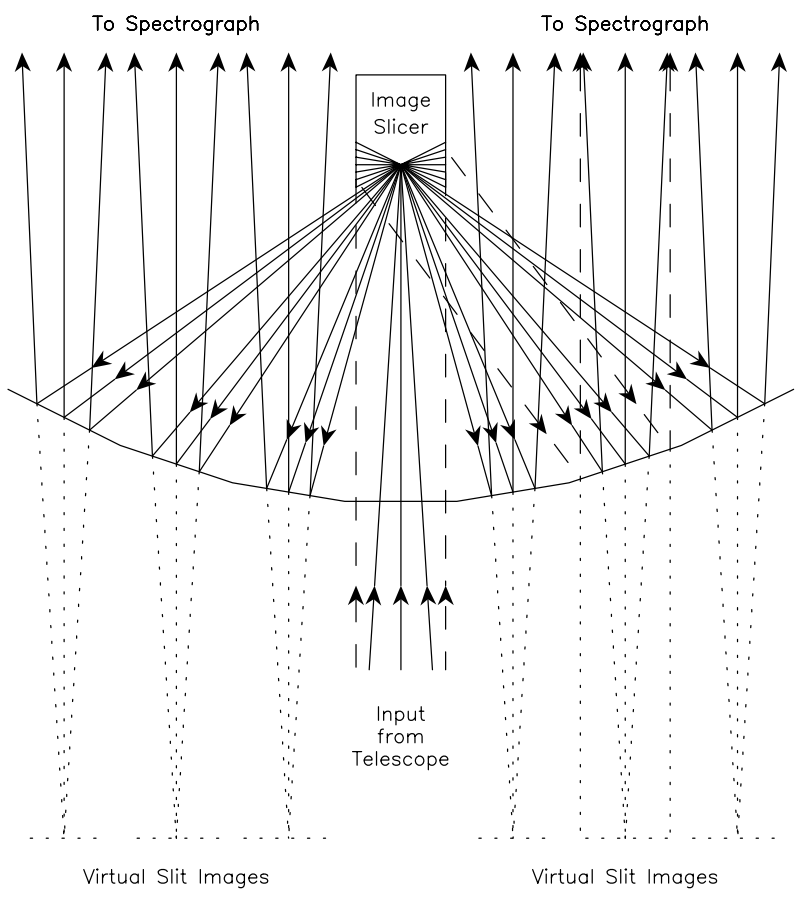

Figure 5-Schematic of the IFU approach used in 3D.

This problem has been addressed by the Astronomical Instrumentation Group at Durham University (Figure 6). They propose using reimaging optics to form separate pupils on each element of the segmented mirror. This means that in NIFS, for example, each $2 \mathrm{~mm}$ long slitlet could form an image only $2 \mathrm{~mm}$ in diameter on the segmented mirror with minimal overlap between segments. The Durham design then uses spherical elements in a pupil mirror array to form a real image of the $f / 16$ slit, and a further array of field mirrors at the real slit image to relocate the system pupil on the grating.

Implementing this design in GNIRS requires large off-axis angles between the optical components, which introduce significant aberrations. By designing the IFU as an integral part of the spectrograph, we can achieve a simpler optical design with better performance (Figure 1).

\subsection{NIFS IFU Implementation}

Details of the NIFS IFU are shown in Figure 7. The $11 \mathrm{~mm} \times 11 \mathrm{~mm}$ imager slicer consists

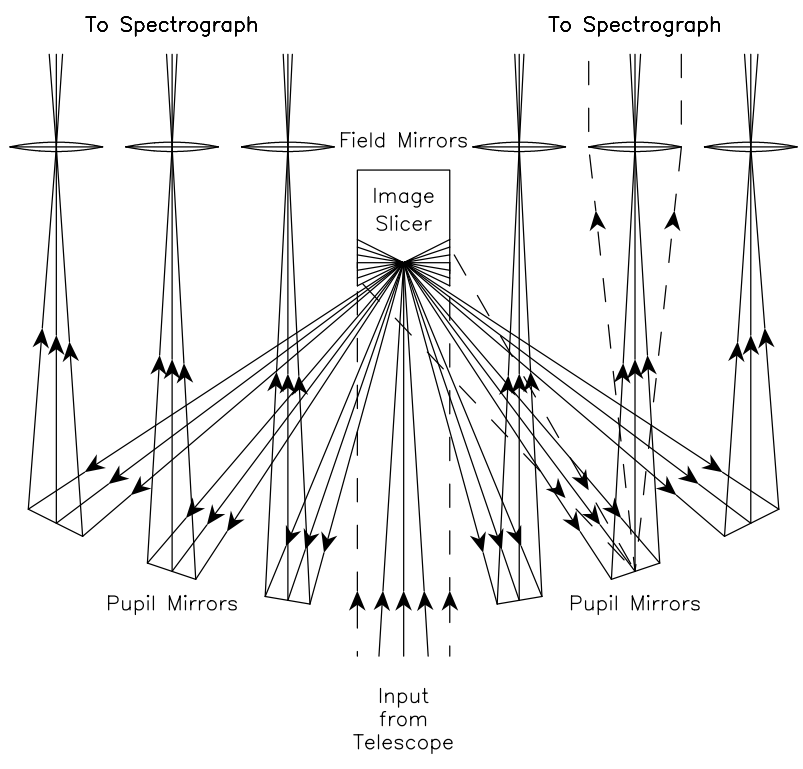

Figure 6-Schematic of the IFU approach proposed by Content (1997).

of 32 flat mirrors, each $0.33 \mathrm{~mm}$ thick. The displacement angles on the image slicer elements are small so that defocus of the $f / 90$ image plane is not significant relative to the diffraction-limited image size (Figure 8). The image slicer will be manufactured by polishing individual glass plates to the required angles, and gluing or clamping the stack of 32 mirrors in a metal mount at the required angles (Figure 9). The $0 \cdot 33 \mathrm{~mm}$ thickness of each slicer element is sufficient to hold an accurate optical polish. The assembled mirror unit will then be gold-coated.

The distance between the image slicer and the pupil mirrors is $120 \mathrm{~mm}$ so that the $1 \cdot 3 \mathrm{~mm}$ diameter $f / 90$ pupils are contained within the $1.6 \mathrm{~mm}$ width of each pupil mirror. The extreme $f / 90$ pupil images are not significantly out of focus so there is no overlap with adjacent pupil images (Figure 10). The pupil mirrors have a focal length of $18.3 \mathrm{~mm}$ so they reimage each slitlet with a focal ratio of $f / 16$ at a distance of $21.6 \mathrm{~mm}$. A flat row of spherical field mirrors is located at this focus. These mirrors have a focal length of $21.6 \mathrm{~mm}$ to feed telecentric beams into the spectrograph. The vertex of each sphere is at the same lateral displacement as the matching pupil mirror, so the field mirrors must be decentred by increasing amounts.

The pupil mirror and field mirror arrays will be either manufactured as micro-mirror arrays, or assembled from individual polished glass mirrors and glued or clamped in place. Micro-mirror arrays will be easier to align, but we are concerned that scattered light from the machined optical surfaces will be problematic, especially for the rejection of terrestrial $\mathrm{OH}$ emission. Individual glass mirrors can be polished with greater accuracy as a larger unit and sliced to the required dimensions (Figures 11 and 12), but these are expected to be more difficult 


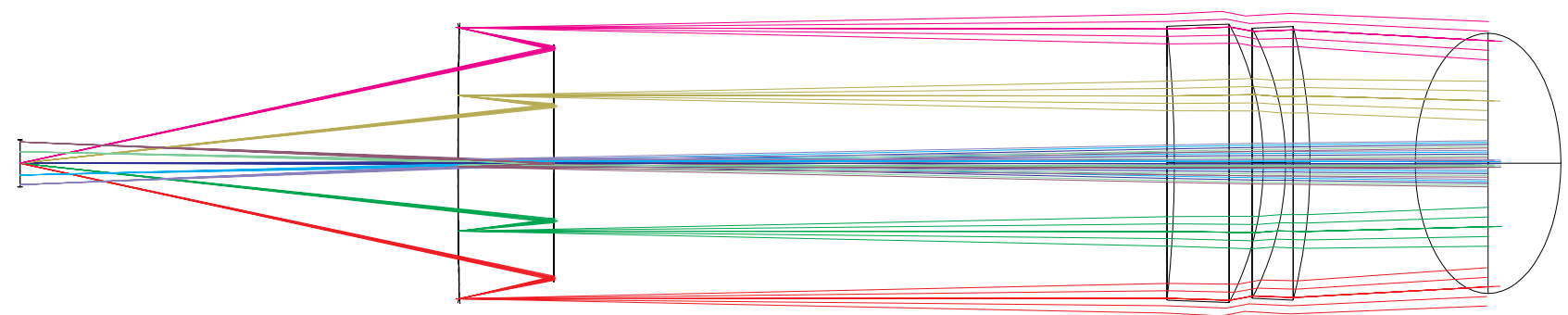

Figure 7-Ray trace of NIFS IFU. The $f / 90$ image slicer is at left. The $f / 90$ pupil is imaged onto the pupil mirror array which reimages the reformatted focal plane at $f / 16$ onto the field mirror array. The field mirror array then feeds telecentric beams into the spectrograph. The field lens and collimator fold mirror of the spectrograph are shown at right.
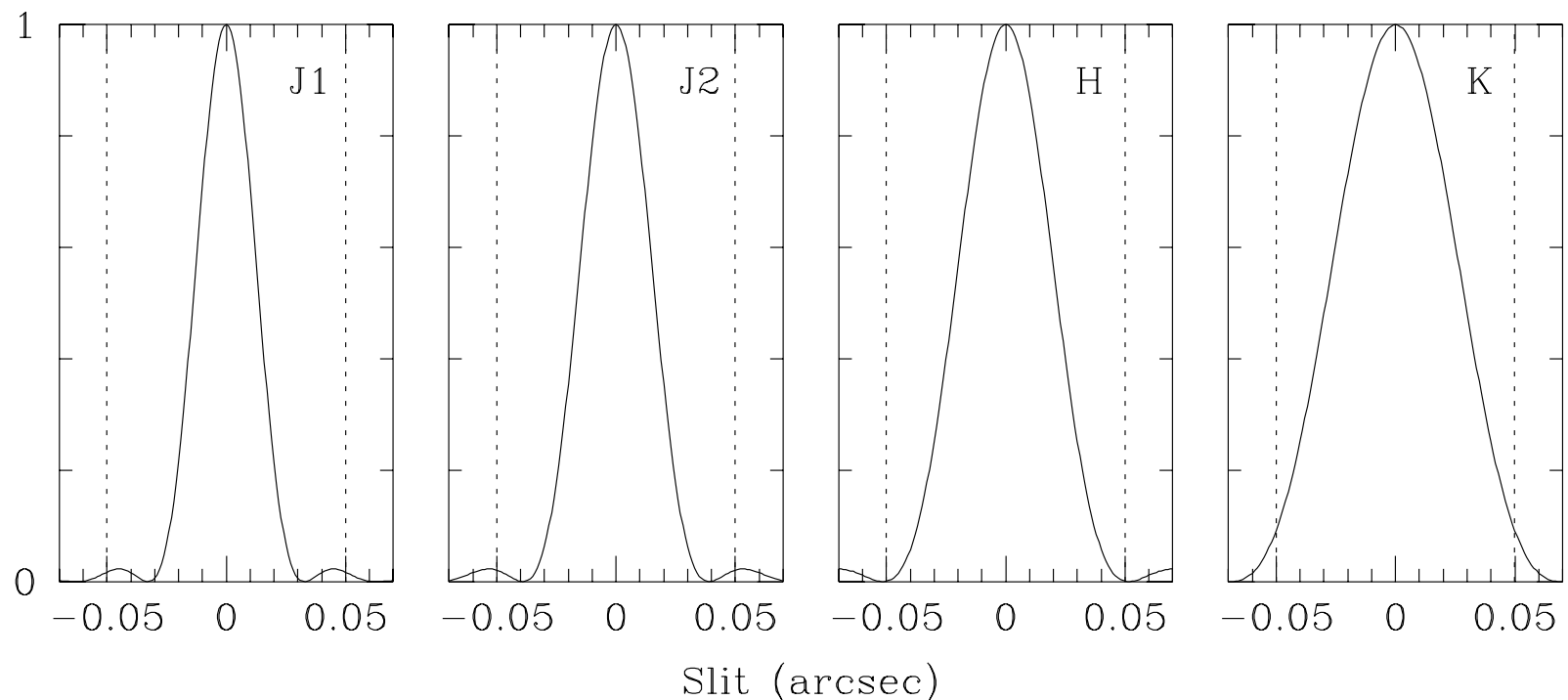

Figure 8-Diffraction profiles at $J 1(1.05 \mu \mathrm{m}), J 2(1.25 \mu \mathrm{m}), H(1.65 \mu \mathrm{m})$ and $K(2.20 \mu \mathrm{m})$ for an object centred in a $0 \cdot 1^{\prime \prime}$ wide slitlet. The slitlet width is indicated by dashed lines.

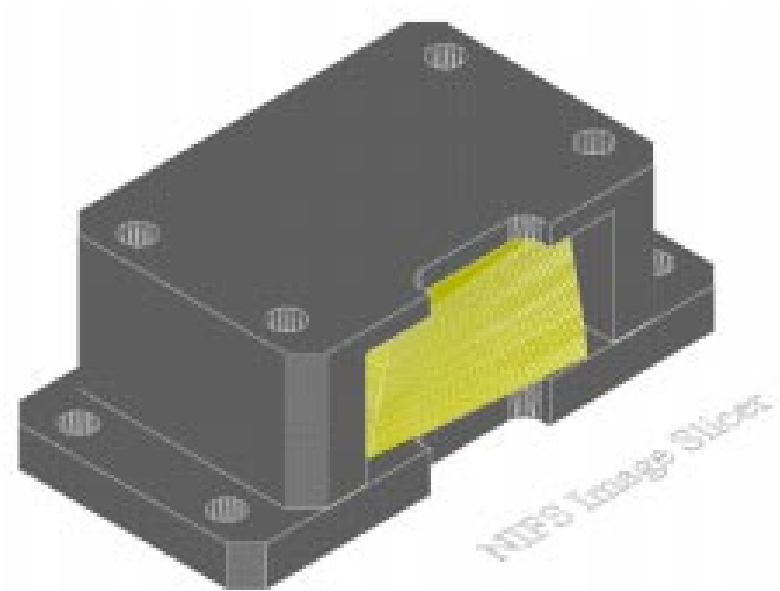

Figure 9 -Image slicer required for the NIFS IFU. The slicer segments have widths of $330 \mu \mathrm{m}$, forming a $10.9 \times 10.9 \mathrm{~mm}$ surface.

to align. A prototype polished glass mirror IFU assembly is shown in Figure 13.

\subsection{Diffraction}

The image diffraction full width at half maximum at $2 \cdot 2 \mu \mathrm{m}$ on Gemini is $\sim 0 \cdot 07^{\prime \prime}$. Consequently, a spectrograph with a $0 \cdot 10^{\prime \prime}$ slit is essentially diffraction-limited in the $K$ band. Slit diffraction causes divergence in the spectral direction of rays through the slit in excess of the geometrical focal ratio; it can be thought of as focal ratio degradation speeding the beam in the spectral direction. This spreads the beam in the IFU in the direction perpendicular to the slicing direction, and can be accommodated by increasing the size of each element of the pupil mirror array in this direction. Since the NIFS spectrograph already accommodates a $64 \mathrm{~mm}$ long reformatted slit, the NIFS collimator optics are quite capable of accepting this diffracted light. Rays outside the geometrical focal ratio then strike the grating outside the $30 \mathrm{~mm}$ diameter geometrical pupil. Passing this diffracted light to the camera requires using a wider grating than would otherwise be the case.

Calculation of the diffraction angle at the slit is complicated because the image slicer is at a focus. A perfect image would have no phase coherence across the slit. In practice, there will be significant phase coherence across the slit due to a combination of the telescope diffraction and seeing effects uncorrected by the $\mathrm{AO}$ system. If we assume full phase coherence, diffraction at $2 \cdot 2 \mu \mathrm{m}$ through a $330 \mu \mathrm{m}$ wide slit produces a first minimum at an angle of $\sim 0 \cdot 38^{\circ}$ to the optical axis. This corresponds to a vertical displacement on the pupil mirror array of 


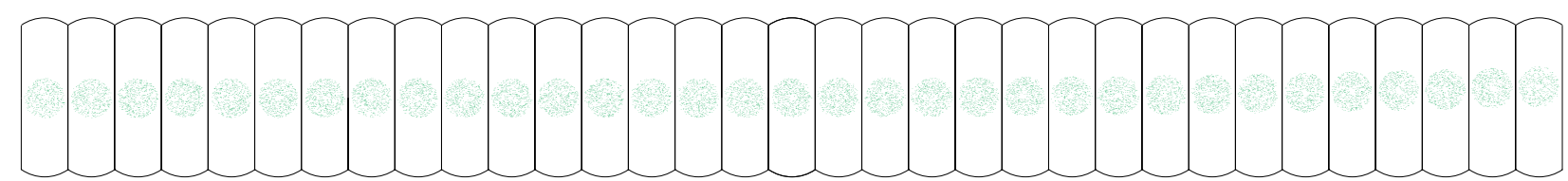

Figure 10-Illumination pattern on the pupil mirrors of the NIFS IFU. All pupil images have a diameter of $1.3 \mathrm{~mm}$, less than the mirror spacing of $1.6 \mathrm{~mm}$.

Table 2. NIFS grating parameters

\begin{tabular}{cccccrr}
\hline Grating & $\begin{array}{c}\lambda_{c} \\
(\mu \mathrm{m})\end{array}$ & $\begin{array}{c}n \\
(\mathrm{l} / \mathrm{mm})\end{array}$ & $\begin{array}{c}\theta_{\text {blaze }} \\
(\mathrm{deg})\end{array}$ & $\begin{array}{c}\theta \\
(\mathrm{deg})\end{array}$ & $\begin{array}{c}R \\
\text { Range } \\
(\mu \mathrm{m})\end{array}$ \\
\hline$J 1$ & $1 \cdot 05$ & 600 & $17 \cdot 5$ & $19 \cdot 0$ & 5090 & $59 \cdot 0$ \\
$J 2$ & $1 \cdot 25$ & 600 & $17 \cdot 5$ & $22 \cdot 8$ & 6100 & $49 \cdot 2$ \\
$H$ & $1 \cdot 65$ & 400 & $13 \cdot 9$ & $20 \cdot 0$ & 5340 & $56 \cdot 2$ \\
$K$ & $2 \cdot 20$ & 300 & $17 \cdot 5$ & $20 \cdot 0$ & 5340 & $56 \cdot 2$ \\
\hline
\end{tabular}

$\pm 0 \cdot 8 \mathrm{~mm}$, which is to be compared to the pupil size of $\pm 0.67 \mathrm{~mm}$. This corresponds to a motion of $\pm 18 \mathrm{~mm}$ in the spectral direction on the grating, which is to be compared to the geometrical grating pupil size of $\pm 15 \mathrm{~mm}$. The decision of how much of this diffracted light to accept will be based on design trade-offs such as the ability to accommodate larger optics and their cost.

\subsection{Grating Choices}

The primary science drivers for NIFS require a twopixel spectral resolving power of $\sim 5000$ to detect black holes in galactic nuclei and study the dynamical evolution of disk galaxies at high redshift, where galactic masses may be lower than for presentday galaxies, and hence the rotational velocities encountered may be lower. A two-pixel resolving power of $\sim 5000$ is also sufficiently high to resolve much of the terrestrial $\mathrm{OH}$ airglow emission which plagues low-resolution near-infrared spectroscopy. Greater sensitivity can be achieved by masking the $\mathrm{OH}$ emission lines recorded in high-resolution spectra in software. With a 2048-pixel detector, nearly complete spectra of the $H$ and $K$ photometric bands can be recorded with a two-pixel spectral resolving power of $R \sim 5340$. Two 2048-pixel regions are required to record all of the $J$ photometric band. Thus all regions of the $0 \cdot 94-2 \cdot 42 \mu \mathrm{m}$ wavelength range within standard photometric passbands can be recorded using just four fixed-angle gratings.

An Ebert angle (i.e. camera-collimator angle) of $30^{\circ}$ is required by the NIFS mechanical configuration. The off-the-shelf gratings listed in Table 2 then produce the required central wavelengths in first order with grating angles of $\sim 20^{\circ}$. A camera focal length of $290 \mathrm{~mm}$ images the full $H$ and $K$ photometric bands onto the detector with a two-pixel resolving power of $5340\left(\Delta v=56 \mathrm{~km} \mathrm{~s}^{-1}\right)$. The $18 \mu \mathrm{m}$ detector pixels map to $0 \cdot 05^{\prime \prime}$ on the sky with a collimator focal length of $480 \mathrm{~mm}$.
The grating wheel will also contain a screen that blocks the spectrograph camera while dark and bias frames are exposed, and a mirror that images the staircase slit onto the detector undispersed. The latter will be used to assist in determining the correct detector focus.

\subsection{Detector Choice}

The primary science drivers for NIFS require maximised spatial coverage and spectral resolution, while making no specific requirement for wavelength coverage beyond $2.5 \mu \mathrm{m}$. NIFS will therefore use the $2048 \times 204818 \mu \mathrm{m}$ pixel $\mathrm{HgCdTe}$ HAWAII-2 array under development by Rockwell. These devices offer the largest available format and best noise performance of contenders in the $1-2.5 \mu \mathrm{m}$ range.

The Rockwell $2048 \times 2048$ array will have been used with SDSU-2 controllers by the University of Hawaii before NIFS is commissioned. NIFS will also use this controller.

\subsection{Optical Performance}

The NIFS optical design has been progressed to a level where it gives 'two-pixel' optical performance. No specific optimisation of the IFU components has yet been performed. Spot diagrams on the detector at $1 \cdot 50,1 \cdot 65$, and $1 \cdot 80 \mu \mathrm{m}$ in an $H$-band spectrum are shown in Figure 14. This figure also shows images of the reformatted slit at these wavelengths. The slit curvature seen in Figure 14 is due to the 'staircase' slit format combined with spectral line curvature induced by the grating.

\subsection{Efficiency}

The NIFS system efficiency has been estimated assuming telescope mirror reflectivities of $95 \%$, gold mirror reflectivities internal to the dewar of $98 \%$, and single-layer $\mathrm{MgF}_{2}$ anti-reflection coatings on all lenses. A grating efficiency of $50 \%$ has been adopted, and the detector quantum efficiency is assumed to 


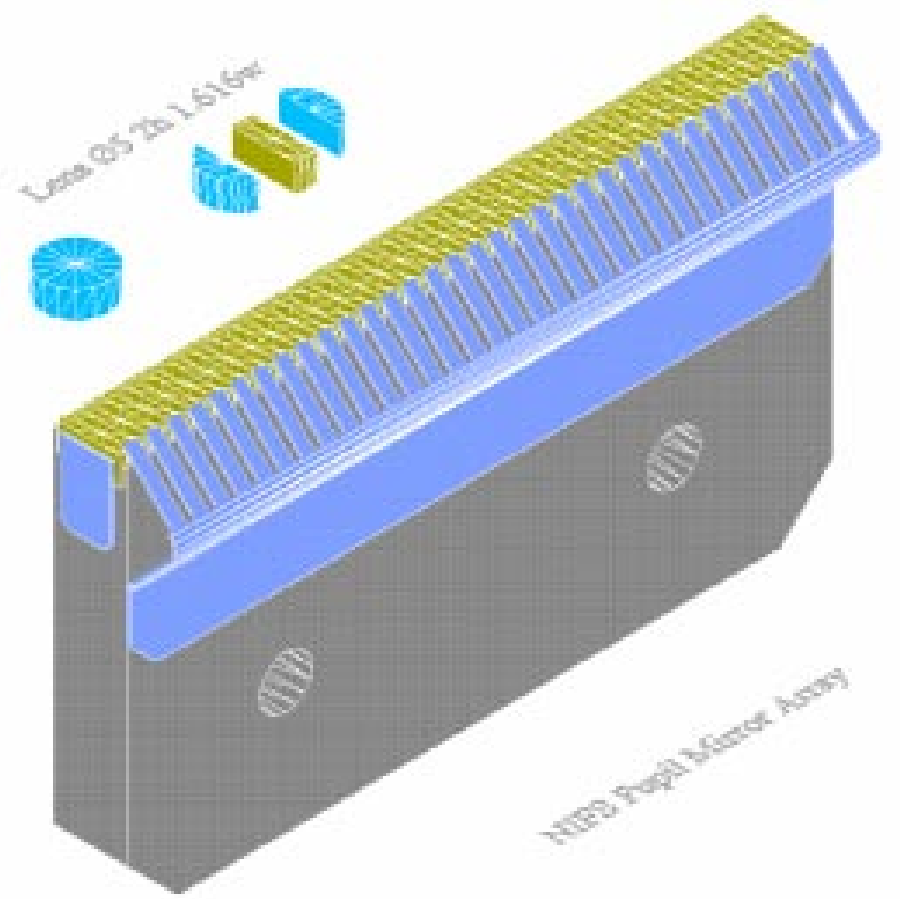

Figure 11-The array of centred pupil mirrors may be constructed by slicing larger glass mirrors.

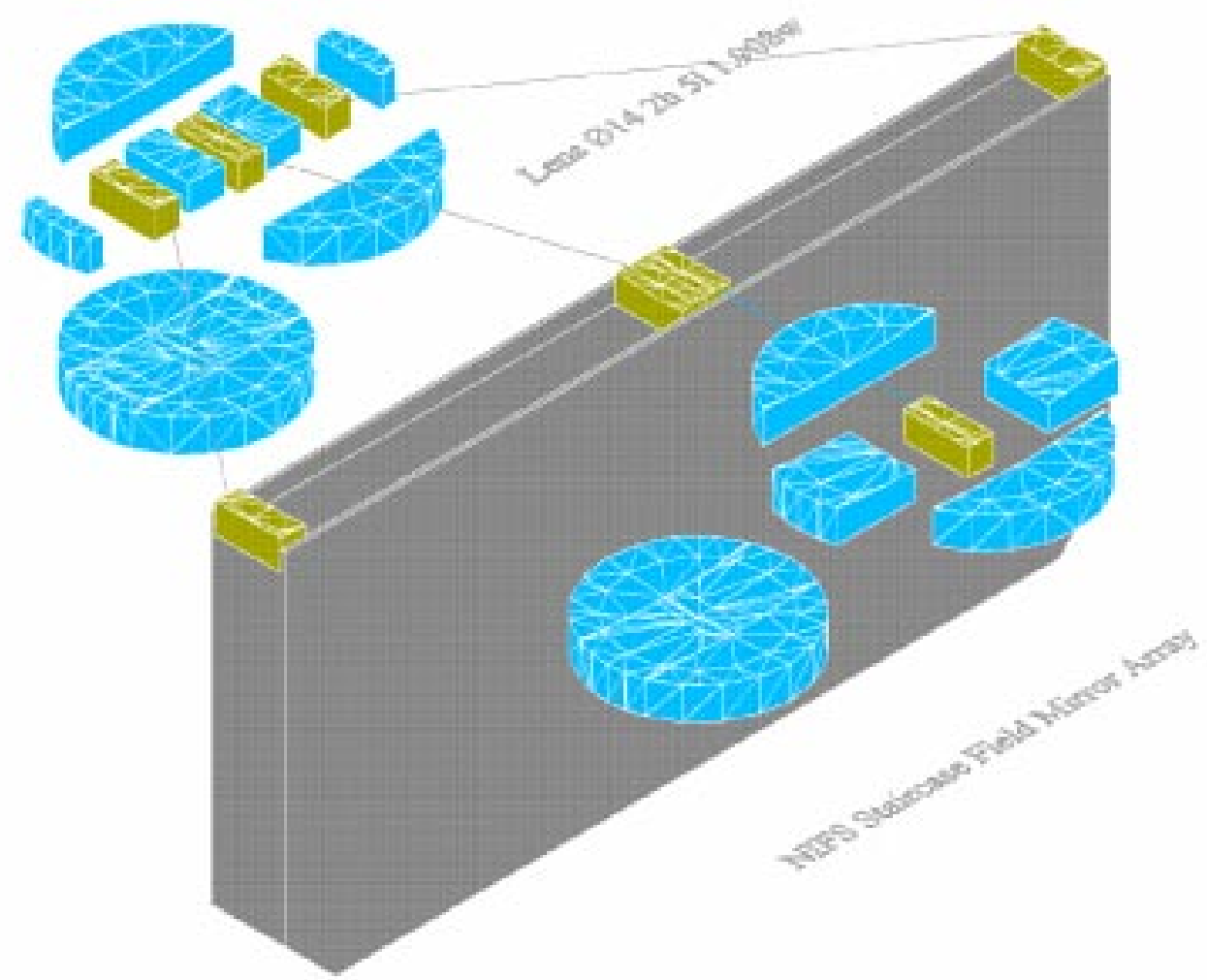

Figure 12-The array of decentred field mirrors may be constructed by slicing decentred sections from larger glass mirrors. 


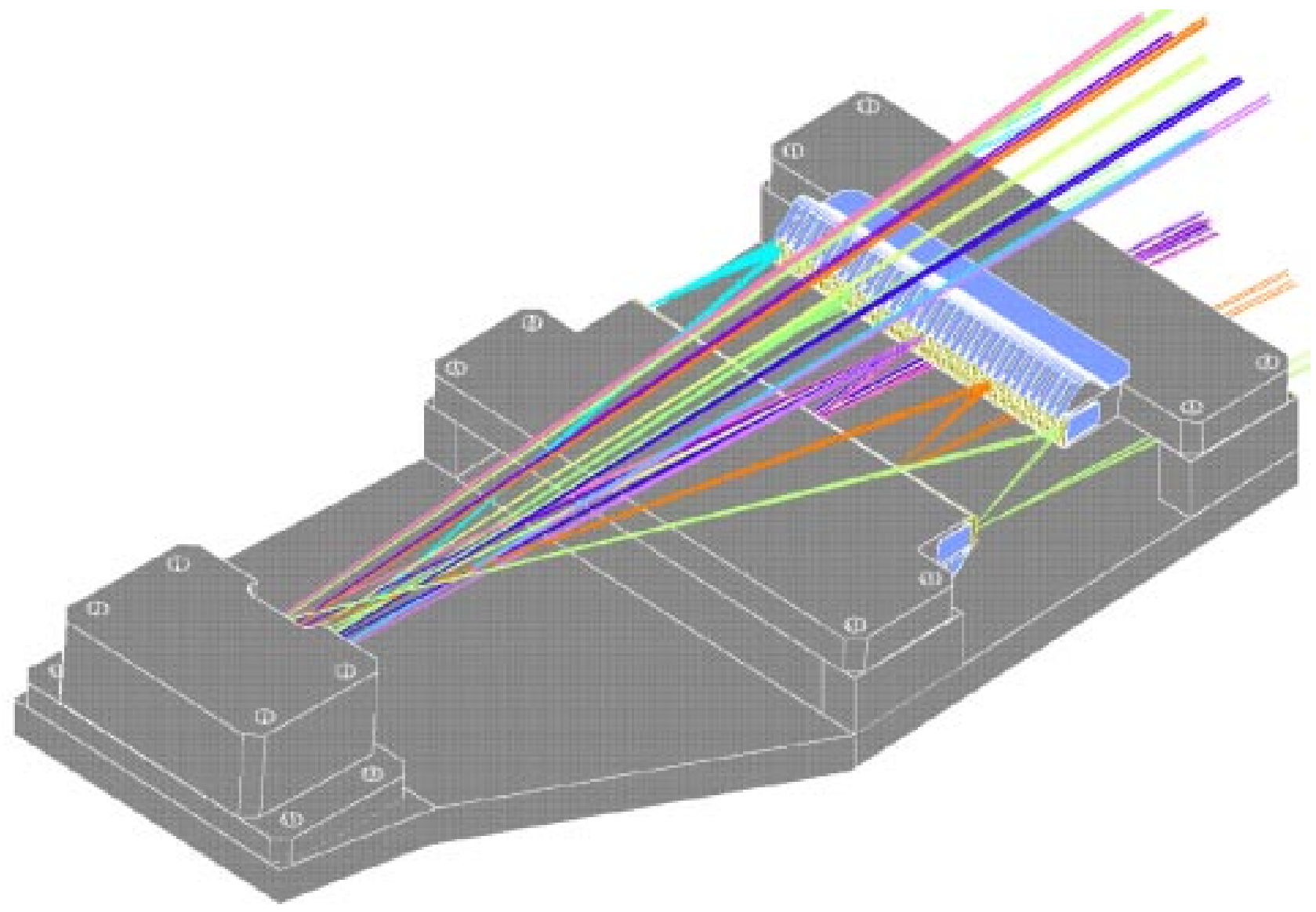

Figure 13-Prototype polished glass mirror IFU assembly showing image slicer at left and pupil and field mirror arrays at right.

be $70 \%$ over the full $0 \cdot 9-2 \cdot 5 \mu \mathrm{m}$ wavelength range. With these assumptions, the NIFS system efficiency, including the Gemini telescope optics but excluding the transmission of the AO system, is $\sim 0 \cdot 16$ $e^{-} /$photon over this wavelength range. The detailed efficiency as a function of wavelength is shown in Figure 15 with and without anti-reflection coatings on the lenses.

\subsection{Sensitivity}

In calculating the NIFS sensitivity, we adopt a read noise of $9 e^{-}$, a dark current of $0.015 e^{-} \mathrm{s}^{-1}$ pixel $^{-1}$, and a maximum frame integration time of $1 \mathrm{hr}$.

Measurements of stellar velocity dispersion in galactic nuclei are limited by the sensitivity for detecting continuum sources. As noted in Section 3.1, the central surface brightness of typical black hole candidate galaxies in the $K$ band is $\sim 13.7$ mag $\operatorname{arcsec}^{-2}$. This will produce $\sim 0 \cdot 12 e^{-} \mathrm{s}^{-1}$ pixel $^{-1}$. The background photocurrent at $2 \cdot 3 \mu \mathrm{m}$ is predicted to be $\sim 0 \cdot 1 e^{-} \mathrm{s}^{-1}$ pixel $^{-1}$ due predominantly to thermal emission from the telescope. In a $1 \mathrm{hr}$ integration, the signal-to-noise ratio (SNR) achieved per spectral and spatial resolution element is $\sim 30$. This is degraded to $\sim 20$ after sky subtraction. This SNR is more than adequate for stellar velocity dispersion measurements, since the intrinsic width of the $\mathrm{CO}$ (2-0) bandhead is $\sim 500 \mathrm{~km} \mathrm{~s}^{-1}$. A similar time will be needed for off-source sky measurement, making the total integration time per object $\sim 2 \mathrm{hr}$.

Atmospheric $\mathrm{OH}$ line emission is the dominant noise source in the $J$ and $H$ bands. Consequently, limiting observations will be restricted to regions between these strong $\mathrm{OH}$ lines. The dominant emission mechanisms in regions between strong $\mathrm{OH}$ lines are not well determined. Non-thermal sky continuum emission or a multitude of weak $\mathrm{OH}$ emission lines may define the limiting sky brightness in the $J$ and $H$ bands. However, it is likely that the limiting detected background will be set by $\mathrm{OH}$ line emission scattered within the dewar. If only $10 \%$ of the total $\mathrm{OH}$ emission entering the dewar is scattered across the detector, the background photocurrent produced will be comparable to the expected detector dark current of $\sim 0 \cdot 015 e^{-} \mathrm{s}^{-1}$ pixel $^{-1}$. The strongest $\mathrm{OH}$ emission is in the $H$ band. A model NIFS $H$-band spectrum showing the expected $\mathrm{OH}$ emission-line photo-currents is presented in Figure 16. These are still sufficiently low that individual integrations will be limited by uncertain factors such as the 


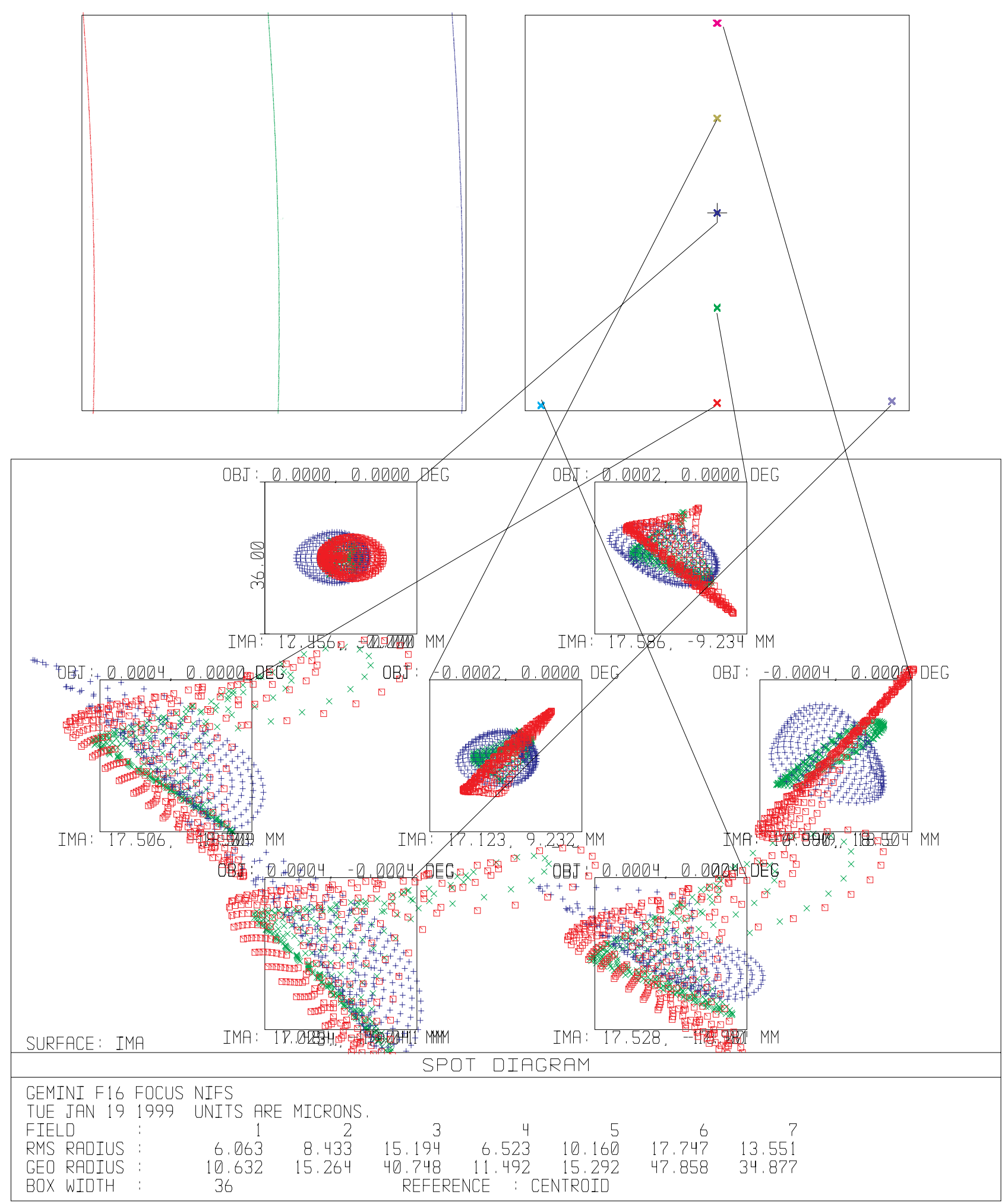

Figure 14-Preliminary spot diagrams at wavelengths of $1.50,1.65$ and $1.80 \mu \mathrm{m}$ on the NIFS detector. The enclosing boxes are $36 \mu \mathrm{m}$ on a side, corresponding to $2 \times 2$ detector pixels. The optical design is yet to be fully optimised. The frame at top right shows the locations on the $f / 90$ image slicer mirror of each field position shown. The frame at top left shows images of the reformatted slit on the detector at the three wavelengths. 


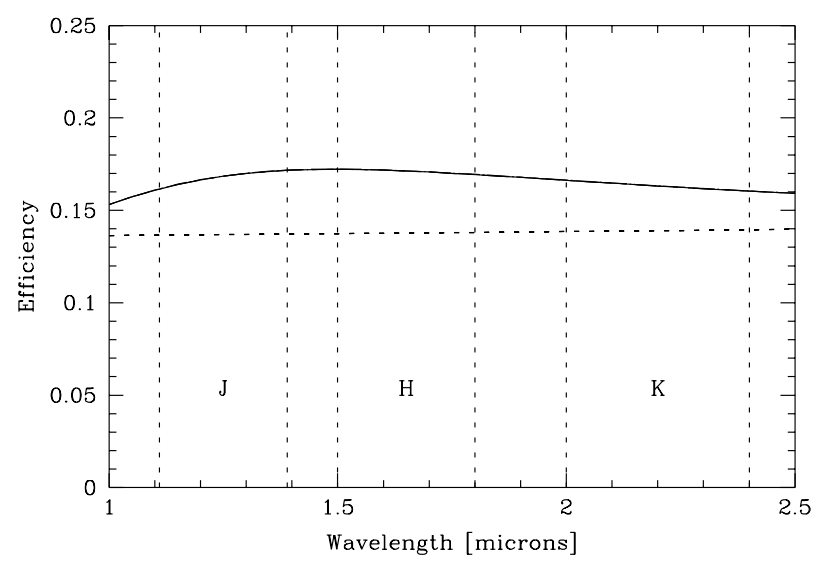

Figure 15-Total NIFS plus Gemini efficiency as a function of wavelength with single-layer $\mathrm{MgF}_{2}$ anti-reflection coatings on all lenses (solid curve) and without anti-reflection coating (dashed line).

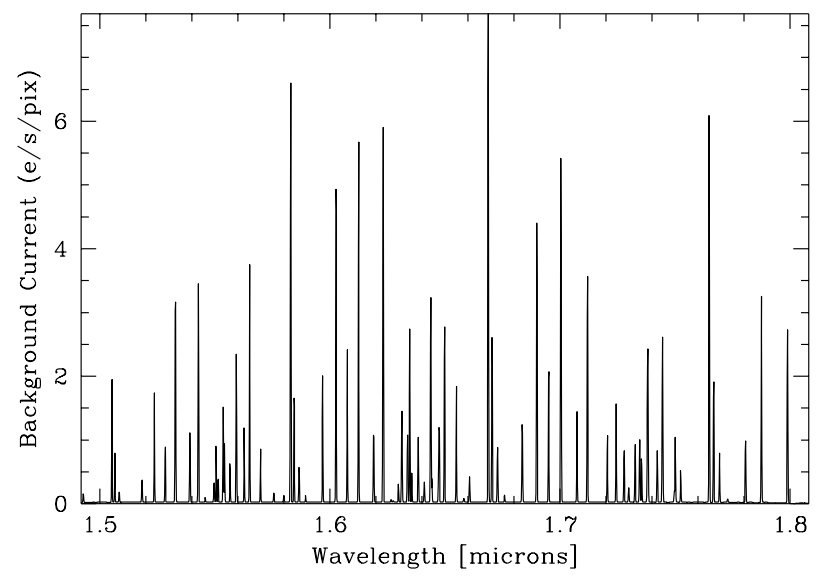

Figure 16-Model $H$-band background spectrum. The spectrum is dominated by terrestrial $\mathrm{OH}$ emission. The continuum between strong $\mathrm{OH}$ lines is uncertain, but will probably be dominated by scattered light.

cosmic-ray event rate and on-chip amplifier glow rather than detector saturation.

For detecting $\mathrm{H} \alpha$ at high redshift, we consider the limiting detectable emission-line flux at wavelengths avoiding the strong $\mathrm{OH}$ emission lines. The $1 \sigma$ noise per pixel in a $1 \mathrm{hr}$ integration is $\sim 13 \cdot 8 \mathrm{e}^{-} \mathrm{pixel}^{-1}$. A $5 \sigma$ detection of a $50 \mathrm{~km} \mathrm{~s}^{-1}$ wide spectral line requires the detection of $\sim 138 e^{-}$, which will be achieved with a line flux of $\sim 7 \cdot 4 \times 10^{-26} \mathrm{~W} \mathrm{~cm}^{-2}$ at $1.31 \mu \mathrm{m}$, corresponding to the wavelength of $\mathrm{H} \alpha$ at $z=1$. The $\mathrm{H} \alpha$ surface brightness through a 1-pixel-long segment of the 2-pixel-wide slit is then $\sim 1.5 \times 10^{-23} \mathrm{~W} \mathrm{~cm}^{-2} \operatorname{arcsec}^{-2}$. Sky measurements will be obtained from the object exposures for sufficiently small objects (typical half-light radius is $\left.\sim 0 \cdot 77^{\prime \prime}\right)$. The effect of sky subtraction on SNR can be offset by averaging two pixels along the slit. In $1 \mathrm{hr}$ of on-source integration on a $z=1$ galaxy with the average $\mathrm{H} \alpha$ surface brightness of $2.5 \times 10^{-23} \mathrm{~W} \mathrm{~cm}^{-2} \operatorname{arcsec}^{-2}$ predicted in Section 3.2 , we would achieve a $7.5 \sigma$ detection of $\mathrm{H} \alpha$ per $0 \cdot 1^{\prime \prime} \times 0 \cdot 1^{\prime \prime}$ spatial element. The integrated spectrum formed by averaging over the $\sim 186$ spatial elements within a typical half-light radius would have a SNR on $\mathrm{H} \alpha$ of $\sim 102$ if the line was only $50 \mathrm{~km} \mathrm{~s}^{-1}$ wide, and a SNR of $\sim 60$ in each peak of a horned profile if approximately one-third of the $\mathrm{H} \alpha$ emission was in the peak.

\section{Timeline}

The NIFS proposal was presented to the Gemini Instrument Forum at its March 1999 meeting. That committee recommended that the NIFS Conceptual Design Study should proceed. The Gemini Science Committee endorsed this recommendation at its April 1999 meeting. Subsequently, the Gemini Board approved the conceptual design study at its May 1999 meeting. The conceptual design study is expected to take until January 2000. At that point, the Gemini Board will decide whether NIFS proceeds to construction. If it does, further design, construction and commissioning is expected to take until the end of 2002, when the instrument will become available to the Gemini community.

\section{Acknowledgments}

Many people have offered comments on the NIFS proposal. The authors would particularly like to acknowledge contributions from Ken Freeman, Matthew Colless, Joss Bland-Hawthorn, Paul Francis, and Karl Glazebrook in developing the science case. Peter McGregor expresses his appreciation to staff at the Institute for Astronomy, University of Hawaii, for open discussions about the status of NIRI.

\section{References}

Bender, R., Kormendy, J., \& Dehnen, W. 1996, ApJ, 464, L123

Bower, G. A., et al. 1998, ApJ, 492, L111

Content, R. 1997, SPIE, 2871, 1295

Devereux, N. A., Becklin, E. E., \& Scoville, N. 1987, ApJ, 312,529

Eckart, A., \& Genzel, R. 1997, MNRAS, 284, 576

Ferrarese, L., Ford, H. C., \& Jaffe, W. 1996, ApJ, 470, 440

Ford, H. C., et al. 1994, ApJ, 435, L27

Franceschini, A., Vercellone, S., \& Fabian, A. C. 1998, MNRAS, 297, 817

Griersmith, D., Hyland, A. R., \& Jones, T. J. 1982, AJ, 87,1106

Kormendy, J., et al. 1996a, ApJ, 459, L57

Kormendy, J., et al. 1996b, ApJ, 473, L91

Kormendy, J., et al. 1997, ApJ, 482, L139

Kormendy, J., Bender, R., Evans, A. S., \& Richstone, D. 1998, AJ, 115, 1823

Kormendy, J., \& Richstone, D. O. 1995, ARA\&A, 33, 581

Lauer, T. R., et al. 1996, ApJ, 471, L79

Lilly, S. J., Tresse, L., Hammer, F., Crampton, D., \& Le Fèvre, O. 1995, ApJ, 455, 108

McCarthy, P. J., et al. 1999, ApJ, 520, 548

Miyoshi, M., et al. 1995, Nature, 373, 127

Offner, A. 1975, Opt. Eng., 14, 130

Sargent, W. L. W., Young, P. J., Boksenberg, A., Shortridge, K., Lynds, C. R., \& Hartwick, F. D. A. 1978, ApJ, 221, 731 
Schade, D., Lilly, S.J., Crampton, D., Hammer, F., Le Fèvre, O., \& Tresse, L. 1995, ApJ, 451, L1

Tresse, L., \& Maddox, S. J. 1998, ApJ, 495, 691

van der Marel, R. P., de Zeeuw, P. T., \& Rix, H. W. 1997, ApJ, 488, 119
Weitzel, L., et al. 1996, A\&AS, 119, 531

Young, T. T., Hodapp, K.-W., Douglass, J., Neill, D., Irwin, E., \& Robertson, L. 1998, SPIE, 3354, 1084 